\title{
Revista Colombiana de

\section{Miocardiopatía de takotsubo desencadenada por la utilización o exposición a drogas de abuso, sustancias químicas o venenos de origen animal}

\author{
Laura Cifuentes ${ }^{\mathrm{a}}$ y Pedro Amariles ${ }^{\mathrm{b}, \mathrm{c}, *}$
}

\author{
a Programa Joven investigadora Colciencias, Grupo de investigación, Promoción y Prevención Farmacéutica, Universidad de \\ Antioquia, Medellín, Colombia \\ b Departamento de Farmacia, Grupo de investigación Promoción y Prevención Farmacéutica, Universidad de Antioquia, Medellín, \\ Colombia \\ c Grupo de investigación en Atención Farmacéutica, Universidad de Granada, Granada, España
}

Recibido el 19 de enero de 2016; aceptado el 25 de mayo de 2016

Disponible en Internet el 11 de agosto de 2016

\author{
PALABRAS CLAVE \\ Miocardiopatía; \\ Takotsubo; \\ Sistema nervioso \\ simpático; \\ Cocaína; \\ Alcohol
}

\begin{abstract}
Resumen
Objetivo: Identificar drogas de abuso, sustancias químicas o venenos de origen animal asociados a cardiopatía de takotsubo.

Método: Revisión estructurada en Pubmed/Medline, para la que se utilizaron los términos: Takotsubo cardiomyopathy, Tako-tsubo cardiomyopathy, stress cardiomyopathy, transient-leftventricular ballooning syndrome, ampulla cardiomyopathy, apical ballooning syndrome, OR broken heart syndrome; together with "drugs abuse", "chemistry substance." "iatrogenic", "drug-induced" OR "induced by". Se seleccionaron artículos publicados entre el 01/01/1990 y $31 / 10 / 2015$, en inglés, francés o español, con acceso a texto completo. Se incluyeron los artículos con reportes de drogas de abuso, sustancias químicas o venenos de origen animal, como posibles desencadenantes de la miocardiopatía. Adicionalmente, se incluyeron las referencias de los artículos seleccionados, valoradas como relevantes.

Resultado: Se recuperaron 852 artículos diferentes y se seleccionaron 23, más 4 referencias relevantes, en las cuales se identificaron 38 casos de miocardiopatía de takotsubo, posiblemente asociados al uso o exposición a drogas de abuso, sustancias químicas o venenos de animales, especialmente, monóxido de carbono $(n=13 ; 34,2 \%)$, cocaína o crack $(n=5,13,2 \%)$, abstinencia al alcohol $(n=5 ; 13,2 \%)$, venenos de origen animal $(n=5 ; 13,2 \%)$ y anfetaminas $(n=4 ; 10,5 \%)$. En total se identificaron 14 sustancias diferentes como posibles desencadenantes de la miocardiopatía en mención.
\end{abstract}

\footnotetext{
* Autor para correspondencia.

Correo electrónico: pedro.amariles@udea.edu.co (P. Amariles).
} 
Conclusión: La miocardiopatía de takotsubo podría ser desencadenada por el uso o la exposición a 14 drogas de abuso, sustancias químicas o venenos de origen animal, las cuales, en la mayoría de los casos, generan sobreestimulación simpática. Por ello se recomienda considerar este tipo de situación en pacientes que presenten este tipo de miocardiopatía, especialmente en quienes no se identifique un desencadenante físico o emocional.

(c) 2016 Sociedad Colombiana de Cardiología y Cirugía Cardiovascular. Publicado por Elsevier España, S.L.U. Este es un artículo Open Access bajo la licencia CC BY-NC-ND (http:// creativecommons.org/licenses/by-nc-nd/4.0/).

\section{KEYWORDS}

Cardiomyopathy;

Takotsubo;

Sympathetic nervous

system;

Cocaine;

Alcohol

\section{Introducción}

El corazón puede sufrir alteraciones asociadas a la utilización de fármacos y otras sustancias, entre ellas, la miocardiopatía por estrés o miocardiopatía de takotsubo. La denominación de miocardiopatía por estrés se debe a que, usualmente, el desencadenante es un suceso o una sustancia que, por diferentes vías, genera estrés físico y/o psicológico. Por su parte, la denominación de miocardiopatía de takotsubo, se refiere a que, en su etapa aguda, el ventrículo izquierdo adopta una forma similar al instrumento usado por los japoneses para atrapar pulpos, conocido como takotsubo $^{1}$

En general, este tipo de cardiopatía se caracteriza por: morfología del ventrículo izquierdo, tiempo de curso transitorio, con recuperación completa en días a semanas, cambios en el electrocardiograma, aumento de los biomarcadores cardiacos y ausencia de estenosis, miocarditis e hipertrofia del miocardio, al igual que de otras condiciones patológicas que pueden explicar la disfunción regional ${ }^{2}$, como enfermedad cerebrovascular o feocromocitoma. Sin embargo, debido a que la miocardiopatía de takotsubo se presenta principalmente en pacientes de edad avanzada y con otros factores de riesgo, en algunos casos, puede coexistir con enfermedad coronaria ${ }^{3}$.

La importancia de identificar de forma acertada esta miocardiopatía se debe a que, en la mayoría de los casos, la recuperación se da por completo sin necesidad de tratamiento farmacológico ${ }^{1}$, pero en otros casos, puede requerirse la utilización de diuréticos, betabloqueadores, inhibidores de la enzima convertidora de angiotensina, bloqueadores de los receptores de la angiotensina-II, estatinas y ácido acetil salicílico ${ }^{4}$. Por su parte, el soporte inotrópico puede agravar la condición del paciente, causando hipotensión marcada, aumento de la probabilidad de utilizar mayor dosis de catecolaminas y, con ello, mayor intensidad de la 
miocardiopatía. Esta situación se explica por la asociación existente entre el aumento en los niveles de estas sustancias con dicha cardiopatía ${ }^{5}$. En este sentido, la miocardiopatía de takotsubo es más frecuente en mujeres posmenopáusicas, en quienes los niveles normales de catecolaminas se ven notoriamente aumentados por situaciones de estrés emocional $^{6}$.

Algunas revisiones estructuradas han mostrado que la utilización de medicamentos con la capacidad de generar, de forma directa o indirecta, un aumento en los niveles de catecolaminas, podrían desencadenar $\mathrm{CTT}^{7}$. En este sentido, desde el punto de vista farmacológico, es posible que el uso o exposición a ciertas drogas de abuso, sustancias químicas y venenos de origen animal que causen aumento en los niveles de catecolaminas, también puedan desencadenar miocardiopatía de takotsubo; sin embargo, no se dispone de una revisión estructurada orientada a identificar casos de miocardiopatía de takotsubo asociados a dichos productos. Por ello, el objetivo de este trabajo fue identificar drogas de abuso, sustancias químicas o venenos de origen animal asociados a la cardiopatía de takotsubo.

\section{Materiales y métodos}

Se hizo una revisión estructurada en la base de datos Medline/PubMed, usando los términos: stress cardiomyopathy AND induced by; takotsubo cardiomyopathy AND induced by; tako-tsubo cardiomyopathy AND induced by; Apical ballooning syndrome AND induced by; transient-leftventricular ballooning syndrome AND induced by; Broken heart syndrome AND induced by; ampulla cardiomyopathy AND induced by; stress cardiomyopathy AND iatrogenic; Stress cardiomyopathy AND drug-induced; takotsubo cardiomyopathy AND iatrogenic; tako-tsubo cardiomyopathy AND iatrogenic; Broken heart syndrome AND iatrogenic; takotsubo cardiomyopathy AND drug-induced; takotsubo cardiomyopathy AND drugs abuse; stress cardiomyopathy AND drugs abuse; stress cardiomyopathy AND chemistry substance. Los artículos publicados entre el 01/01/1990 y el $31 / 10 / 2015$, en inglés, francés o español, con acceso a texto completo. En la revisión se incluyeron los artículos con reportes de drogas de abuso y sustancias químicas o venenos de origen animal (mordeduras o picaduras) como posibles desencadenantes de miocardiopatía de takotsubo. Adicionalmente, se incluyeron las referencias de los artículos seleccionados, valoradas como relevantes. Por su parte, se excluyeron los artículos sin una descripción clara de una sustancia como desencadenante de miocardiopatía de takotsubo y las cartas con comentarios de casos ya identificados. De cada caso se recopiló la edad, género del paciente y la sustancia desencadenante o asociada a la miocardiopatía de takotsubo.

\section{Resultados}

En la búsqueda en la base de datos Medline/PubMed se identificaron en total 2.599 artículos, de los cuales 1.747 referencias se repiten (tabla 1 ). De las 852 referencias diferentes, se excluyeron $829 ; 2$ por no presentar una relación de causalidad clara entre la sustancia y el desarrollo de miocardiopatía de takotsubo (uso de suplemento dietario ${ }^{8}$, y
Tabla 1 Número de referencias recuperadas por la combinación de términos de búsqueda

\begin{tabular}{lc}
\hline Términos de búsqueda & $\begin{array}{l}\text { Número de } \\
\text { referencias } \\
(\mathrm{n}=2.599)\end{array}$ \\
\hline Stress cardiomyopathy AND induced by & 814 \\
Takotsubo cardiomyopathy AND induced by & 406 \\
Tako-tsubo cardiomyopathy AND induced by & 414 \\
Apical ballooning syndrome AND induced by & 418 \\
Transient-left-ventricular ballooning & 35 \\
$\quad$ syndrome AND induced by & \\
Broken heart syndrome AND induced by & 408 \\
Ampulla cardiomyopathy AND induced by & 7 \\
Stress cardiomyopathy AND iatrogenic & 20 \\
Stress cardiomyopathy AND drug-induced & 10 \\
Takotsubo cardiomyopathy AND iatrogenic & 18 \\
Tako-tsubo cardiomyopathy AND iatrogenic & 18 \\
Broken heart syndrome AND iatrogenic & 18 \\
Takotsubo cardiomyopathy AND & 2 \\
$\quad$ drug-induced & \\
Takotsubo cardiomyopathy AND drugs abuse & 4 \\
Stress cardiomyopathy AND drugs abuse & 4 \\
Stress cardiomyopathy AND chemistry & 3 \\
substance & \\
\hline
\end{tabular}

picadura de avispa ${ }^{9}$ ) y 5 cartas al editor con comentarios de casos ya reportados ${ }^{10-14}$. Adicionalmente, se excluyeron 48 artículos con conceptos teóricos relacionados con la miocardiopatía de takotsubo (fig. 1). Por tanto, para este trabajo se seleccionaron y revisaron 23 referencias con reportes de casos. Adicionalmente, de las referencias de los artículos incluidos se identificaron 4 relevantes para la revisión, para un total de 27 referencias incluidas. De este grupo, un artículo reporta $12 \operatorname{casos}^{15}$ y 26 artículos reportan un caso ${ }^{16-41}$ (fig. 1).

En los 27 artículos incluidos, se identificaron 38 casos de miocardiopatía de takotsubo en pacientes con una edad media de 43 años (rango de 4 a 83 años), y $24(63,2 \%)$ de ellos en mujeres (tabla 2). En los 38 casos se identificaron 14 tipos de sustancias diferentes que, por su uso o por retiro repentino, se asociaron con la aparición de miocardiopatía de takotsubo (tabla 2):

- Intoxicación por monóxido de carbono $(n=13$ casos, $34,2 \%)^{15,16}$.

- Consumo de cocaína o crack ( $n=5$ casos, $13,2 \%)^{17-21}$.

- Abstinencia al alcohol ( $n=5$ casos, $13,2 \%)^{22-26}$.

- Venenos de origen animal $(n=5$ casos, $13,2 \%)$ : picadura de medusa $(n=2)^{27,28}$, picadura de araña "viuda negra" $(n=1)^{29}$, mordedura de serpiente $(n=1)^{30}$ y picadura de escorpión $(n=1)^{31}$.

- Consumo de anfetaminas ( $n=4$ casos, $10,5 \%)^{32-35}$.

- Consumo de sustancias naturales ( $n=2$ casos, $5,2 \%$ ): hongo Psilocybe semilanceata $(n=1)^{36}$ y marihuana $(n=1)^{37}$.

- Abstinencia a la heroína $(n=1 ; 2,6 \%)^{38}$.

- Veneno sintético compuesto por carbamatos/piretroides $(n=1 ; 2,6 \%)^{39}$.

- Intoxicación con cloroquina $(n=1 ; 2,6 \%)^{40}$ y

- Consumo de bebida energizante $(n=1 ; 2,6 \%)^{41}$. 
Tabla 2 Reporte de 38 casos de cardiopatía de takotsubo asociados a la utilización o exposición de drogas de abuso, sustancias químicas o venenos de origen animal

\begin{tabular}{|c|c|c|c|c|c|}
\hline Ref. & Título & Sexo & Edad & Sustancia & Información adicional \\
\hline 15 & $\begin{array}{l}\text { Monóxido de } \\
\text { carbono induce } \\
\text { miocardiopatía }\end{array}$ & $\begin{array}{l}F \\
F \\
F \\
F \\
F \\
F \\
M \\
M \\
F \\
M \\
F \\
M\end{array}$ & $\begin{array}{l}18 \\
26 \\
37 \\
33 \\
75 \\
36 \\
42 \\
22 \\
83 \\
69 \\
43 \\
79\end{array}$ & $\begin{array}{l}\text { Monóxido de } \\
\text { carbono }\end{array}$ & $\begin{array}{l}\text { Estudio retrospectivo que } \\
\text { concluye que } 12 \text { personas } \\
\text { presentaron miocardiopatía } \\
\text { de takotsubo asociada a } \\
\text { intoxicación con monóxido de } \\
\text { carbono }\end{array}$ \\
\hline 16 & $\begin{array}{l}\text { Miocardiopatía transitoria } \\
\text { secundaria a intoxicación } \\
\text { con monóxido de carbono }\end{array}$ & M & 4 & Monóxido de carbono & $\begin{array}{l}\text { Cardiopatía asociada a } \\
\text { convulsiones tónico-clónicas de } \\
\text { corta duración y de resolución } \\
\text { espontánea }\end{array}$ \\
\hline 17 & $\begin{array}{l}\text { "Balonamiento" apical } \\
\text { transitorio del ventrículo } \\
\text { izquierdo después del } \\
\text { consumo excesivo de } \\
\text { cocaína }\end{array}$ & $\mathrm{F}$ & 56 & Cocaína & $\begin{array}{l}\text { Presentes opresión en el pecho } \\
\text { y falta de aliento, horas } \\
\text { después de consumir cocaína }\end{array}$ \\
\hline 18 & $\begin{array}{l}\text { Miocardiopatía de takotsubo } \\
\text { presentada como infarto de } \\
\text { miocardio sin elevación del } \\
\text { segmento ST y exacerbación } \\
\text { del asma debido al consumo } \\
\text { de cocaína }\end{array}$ & M & 53 & Cocaína & $\begin{array}{l}\text { Exacerbación grave de asma. } \\
\text { Tiene un consumo pesado de } \\
\text { cocaína }\end{array}$ \\
\hline 19 & $\begin{array}{l}\text { Discinesia apical transitoria } \\
\text { después del consumo de } \\
\text { cocaína: relación entre la } \\
\text { cardiotoxicidad por } \\
\text { catecolaminas y la patología }\end{array}$ & $\mathrm{F}$ & 54 & Cocaína ("crack") & $\begin{array}{l}\text { Dolor en el pecho. Ha } \\
\text { aumentado la ingesta de } \\
\text { "crack" en los últimos meses. } \\
\text { El último consumo ocurrió } 2 \text { a } 3 \\
\text { horas previas a los síntomas }\end{array}$ \\
\hline 20 & $\begin{array}{l}\text { Miocardiopatía de } \\
\text { takotsubo posiblemente por } \\
\text { cocaína }\end{array}$ & $\mathrm{F}$ & 58 & Cocaína ("crack") & $\begin{array}{l}\text { Dolor severo del lado izquierdo } \\
\text { del pecho. Es consumidora } \\
\text { regular de "crack". La última } \\
\text { ingesta fue a menos de } 24 \\
\text { horas de los síntomas }\end{array}$ \\
\hline 21 & $\begin{array}{l}\text { Miocardiopatía reversible } \\
\text { asociada a consumo por } \\
\text { cocaína }\end{array}$ & $\mathrm{F}$ & 34 & Cocaína ("crack") & $\begin{array}{l}\text { El paciente fumó "crack" } \\
\text { durante todo un día, previo a } \\
\text { los síntomas. Aunque en } 1989 \\
\text { aún no se conocía la } \\
\text { cardiopatía de takotsubo, la } \\
\text { descripción y clínica del caso } \\
\text { fue muy compatible con este } \\
\text { tipo de alteración }\end{array}$ \\
\hline 22 & $\begin{array}{l}\text { Mlocardiopatía takotsubo } \\
\text { desencadenada por } \\
\text { abstinencia de alcohol }\end{array}$ & M & 56 & $\begin{array}{l}\text { Abstinencia de } \\
\text { alcohol }\end{array}$ & $\begin{array}{l}\text { Confusión, astenia severa, } \\
\text { anorexia y temblor de las } \\
\text { manos. } 5 \text { días antes dejó de } \\
\text { consumir alcohol }\end{array}$ \\
\hline 23 & $\begin{array}{l}\text { Miocardiopatía de takotsubo } \\
\text { relacionada con la } \\
\text { abstinencia de alcohol }\end{array}$ & $\mathrm{F}$ & 41 & $\begin{array}{l}\text { Abstinencia de } \\
\text { alcohol }\end{array}$ & $\begin{array}{l}\text { Dolor epigástrico, náusea y } \\
\text { vómito } 24-48 \text { horas después de } \\
\text { interrumpir el consumo de } \\
\text { alcohol }\end{array}$ \\
\hline 24 & $\begin{array}{l}\text { Abstinencia de alcohol } \\
\text { induce miocardiopatía de } \\
\text { takotsubo }\end{array}$ & $\mathrm{F}$ & 57 & $\begin{array}{l}\text { Abstinencia de } \\
\text { alcohol }\end{array}$ & $\begin{array}{l}\text { Agitación, bradipnea e hipoxia. } \\
\text { Por motivo de intervención } \\
\text { quirúrgica suspende el } \\
\text { consumo de alcohol }\end{array}$ \\
\hline
\end{tabular}


Tabla 2 (continuación)

\begin{tabular}{llllll}
\hline Ref. & Título & Sexo & Edad & Sustancia & Información adicional \\
\hline 25 & $\begin{array}{l}\text { Un caso atípico de } \\
\text { "miocardiopatía } \\
\text { takotsubo" durante la }\end{array}$ & M & 64 & $\begin{array}{l}\text { Abstinencia de } \\
\text { alcohol }\end{array}$ & $\begin{array}{l}\text { Cinco días después de } \\
\text { suspender el consumo de } \\
\text { abstinencia de alcohol: } \\
\text { anomalía transitoria en el }\end{array}$ \\
& & & & $\begin{array}{l}\text { alcohol experimenta paro } \\
\text { cardiorrespiratorio }\end{array}$
\end{tabular}

movimiento de

ventricular izquierda y

elevación notable en el segmento ST

Dos casos de síndrome de discinesia apical oculta en

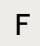

una miocardiopatía

hipertrófica apical

Picadura de medusa

mediterránea induce

miocardiopatía takotsubo

28

Síndrome Irukandji,

catecolaminas y

miocardiopatía por estrés

medio ventricular

29

Miocardiopatía transitoria

inversa (takotsubo) después

de la picadura de araña en

Grecia

Miocardiopatía takotsubo en una víctima de picadura de serpiente: reporte de caso

Primera descripción de un caso de envenenamiento grave por picadura de escorpión: posible miocardiopatía de takotsubo

32 Shock cardiogénico después del uso de fluoro anfetamina confirmado por su presencia en sangre y orina

"Miocardiopatía basal por estrés inducida por catecolaminas exógenas en adultos jóvenes

34 Metanfetamina asociada a disfunción aguda del ventrículo izquierdo (variación de miocardiopatía inducida por estrés)

35 Síndrome del

"balonamiento" apical invertido del ventrículo izquierdo (miocardiopatía takotsubo) en una mujer joven en el ajuste del uso de anfetamina
F $56 \quad$ Mordedura de serpiente

M

7

Veneno de escorpión (Tityus serrulatus)

M

18

4-Fluoroanfetamina (4-FA)

F

36

Metanfetamina oral

$\mathrm{F}$

42

Metanfetamina

$\mathrm{F}$

25

Anfetaminas
Confusión, agitación y falta de aire. 3 días antes había suspendido el consumo de alcohol

Intensa sensación de picazón y posterior pérdida el conocimiento Dolor, agitación y palpitaciones 2 horas después de ser picado por una medusa Carukia barnesi

Una hora y media después de la picadura de araña, el pacientes experimentó náusea, temblor generalizado, sudoración y calambres en las extremidades inferiores

Anomalías en el electrocardiograma cuatro días después de la mordedura de serpiente

Dos horas después de la picadura del escorpión Tityus serrulatus, la pacientes presentó vómito, sudoración profusa y dificultad respiratoria Vómitos, dificultad para respirar, opresión en el pecho, y estado mental alterado. Un examen de orina y sangre demostró la presencia de la anfetamina

Disnea. Dos horas antes ingirió $0,2 \mathrm{~g}$ de metanfetamina oral

Considerado como el primer caso reportado de cardiopatía de takotsubo asociado al abuso de metanfetamina

La paciente manifiesta "falta de aliento"' horas después de inhalar anfetaminas 
Tabla 2 (continuación)

\begin{tabular}{|c|c|c|c|c|c|}
\hline Ref. & Título & Sexo & Edad & Sustancia & Información adicional \\
\hline 36 & $\begin{array}{l}\text { Alteraciones de la } \\
\text { contractilidad regional } \\
\text { apical que evoca a la } \\
\text { miocardiopatía takotsubo } \\
\text { seguido del consumo de } \\
\text { hongos psicoactivos }\end{array}$ & M & 17 & $\begin{array}{l}\text { Hongo Psilocybe } \\
\text { semilanceata }\end{array}$ & $\begin{array}{l}\text { Dolor en el pecho y disnea } \\
\text { luego de la ingesta del hongo } \\
\text { Psilocybe semilanceata }\end{array}$ \\
\hline 37 & $\begin{array}{l}\text { Mioardiopatía ventricular } \\
\text { como una variante de } \\
\text { takotsubo asociada con el } \\
\text { síndrome de hiperémesis } \\
\text { cannabinoide: reporte de } \\
\text { un caso }\end{array}$ & $\mathrm{F}$ & 32 & Marihuana & $\begin{array}{l}\text { Después de un período de } \\
\text { abstinencia experimenta dolor } \\
\text { epigástrico, náusea y vómito al } \\
\text { consumir marihuana }\end{array}$ \\
\hline 38 & $\begin{array}{l}\text { Caso de abstinencia de } \\
\text { heroína que termina en } \\
\text { miocardiopatía takotsubo }\end{array}$ & M & 40 & $\begin{array}{l}\text { Abstinencia a la } \\
\text { heroína }\end{array}$ & $\begin{array}{l}\text { Náuseas, vómitos, nerviosismo } \\
\text { y palpitaciones. Está en } \\
\text { proceso de desintoxicación de } \\
\text { heroína }\end{array}$ \\
\hline 39 & $\begin{array}{l}\text { Miocardiopatía takotsubo } \\
\text { relacionada con } \\
\text { intoxicación por carbamato } \\
\text { y piretroide }\end{array}$ & $\mathrm{F}$ & 80 & $\begin{array}{l}\text { Veneno químico } \\
\text { (insecticida) }\end{array}$ & $\begin{array}{l}\text { En un intento de suicidio } \\
\text { ingiere un pesticida que } \\
\text { contiene } 12 \% \text { de metomilo, } \\
1,5 \% \text { de alfa-cipermetrina y } \\
86,5 \% \text { de otros disolventes }\end{array}$ \\
\hline 40 & $\begin{array}{l}\text { Cardiomiopatía reversible } \\
\text { takotsubo asociada a la } \\
\text { intoxicación por cloroquina }\end{array}$ & $\mathrm{F}$ & 35 & Cloroquina & $\begin{array}{l}\text { La paciente ingirió } 7 \text { gramos de } \\
\text { cloroquina y murió }\end{array}$ \\
\hline 41 & $\begin{array}{l}\text { Miocardiopatía takotsubo } \\
\text { reversible asociada con el } \\
\text { consumo de una bebida } \\
\text { energética }\end{array}$ & M & 24 & Bebida energética & $\begin{array}{l}\text { Palpitaciones y dolor en el } \\
\text { pecho que se experimentan } \\
\text { poco tiempo después de ingerir } \\
\text { una bebida energética. }\end{array}$ \\
\hline
\end{tabular}

* Artículos con información adicional generada por cartas al editor ${ }^{10,13}$

\section{Discusión}

En esta revisión se identificaron 38 casos de miocardiopatía de takotsubo asociados al uso o exposición a 15 drogas de abuso, sustancias químicas o venenos de origen animal, en los cuales se descartó como desencadenante el estado de estrés emocional. En la mayoría se estableció una relación entre la miocardiopatía de takotsubo y los efectos de dichas sustancias, la respuesta física (en casos de abstinencia) y los niveles de catecolaminas presentes en la circulación, hecho que refuerza la hipótesis sobre el efecto del aumento, directo o indirecto, de los niveles de catecolaminas ${ }^{7}$.

El uso de sustancias psicoactivas ha hecho parte de la historia del hombre, quien las ha asociado a diversas prácticas, desde religiosas y curativas hasta de defensa y supervivencia. Sin embargo, el uso y abuso de dichos productos es un problema de salud pública principalmente para la población adulta-joven, en quienes su consumo es mayor. En este contexto, es importante destacar, además de las consecuencias sociales, los efectos cardiacos de algunas de estas sustancias, asociadas a la generación de estados hiperadrenérgicos ${ }^{42}$.

En general, la información generada con diversos casos de miocardiopatía de takotsubo ha permitido establecer el papel fisiopatológico relevante del aumento de los niveles catecolaminas presentes en la circulación. Así, el incremento de estas sustancias puede causar daño directo al miocardio por la activación de los receptores beta-1 y beta-2, mediada por la estimulación de la proteína $G(\mathrm{Gs})$, fenómeno que puede generar estrés oxidativo cardiotóxico a través del adenosín monofosfato cíclico (CAMP) dependiente de la proteína quinasa A (PKA), sobrecarga de calcio $y$, finalmente, muerte celular. Esta alteración inotrópica se evidencia más en el miocardio apical, debido a que en esta zona hay mayor densidad de receptores beta ${ }^{43}$.

En este trabajo, similar a lo que se estableció en revisiones estructuradas de casos de miocardiopatía de takotsubo por medicamentos ${ }^{7}$, en la mayoría de los 38 casos, el uso o exposición a drogas de abuso, sustancias químicas o venenos de origen animal y la presentación de miocardiopatía de takotsubo, podría explicarse por un aumento en los niveles de catecolaminas, tal como se comenta a continuación:

- Cardiopatía de takotsubo por monóxido de carbono (CO) $(13 / 38 \text { casos, equivalente al } 34,2 \%)^{15,16}$ : aunque, el mecanismo principal de toxicidad por CO se atribuye a la hipoxia isquémica secundaria a la hipoxemia, se han identificado otras posibles explicaciones, entre ellas: a) Aumento en la producción neuronal en el sistema nervioso simpático y, con ello, incremento en los niveles de las catecolaminas epinefrina y norepinefrina; b) Estimulación de la actividad del sistema nervioso simpático y, con 


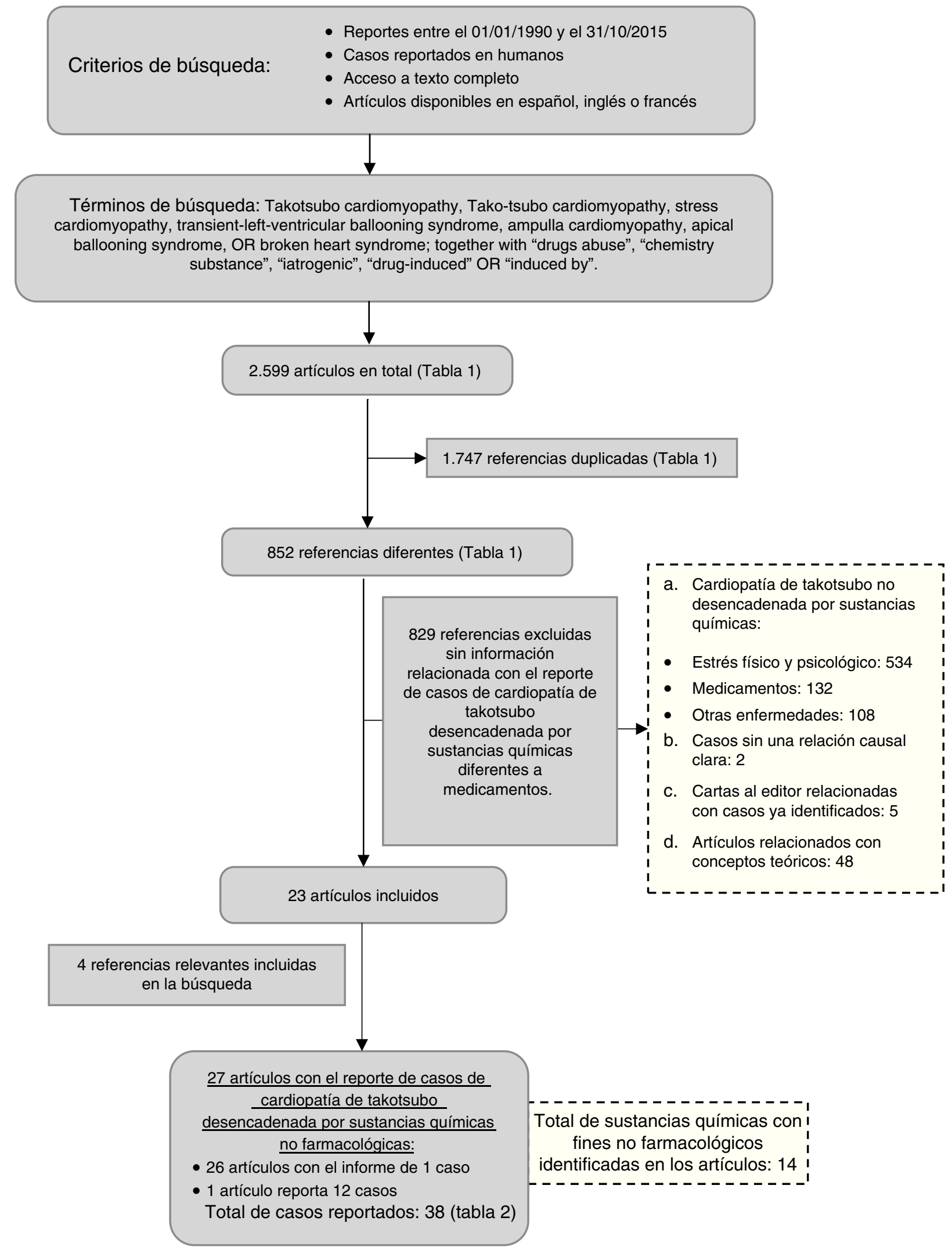

Figura 1 Diagrama de flujo del resultado de la revisión estructurada. 
ello, aumento del gasto cardiaco y de la presión arterial; c) Producción de radicales libres en la lesión por isquemiareperfusión; y d) Aturdimiento miocárdico, posiblemente por el aumento de catecolaminas ${ }^{15,16}$.

- Cardiopatía de takotsubo por cocaína (5/38 casos equivalente al $13,2 \%)^{17-21}$ : la cocaína bloquea la recaptación presináptica de dopamina y norepinefrina, aumentando la concentración de estas aminas en la hendidura sináptica. En general, los efectos psico-estimulantes de la cocaína se atribuyen a un aumento en la neurotransmisión de dopamina; mientras que los efectos cardiovasculares se deben a un mayor estado simpaticomimético. La cocaína tiene acción directa o indirecta sobre el corazón y los vasos sanguíneos, mediada por estimulación de receptores alfa y beta. La estimulación de los receptores alfa-1 adrenérgicos causa vasoconstricción arterial, seguida de la elevación en la presión arterial; por su parte, la estimulación de los receptores beta-adrenérgicos genera un aumento en la frecuencia, contractilidad y demanda de oxígeno por el corazón. La vasoconstricción se amplifica por la inhibición de la síntesis de óxido nítrico y por el aumento de la formación de endotelina-1. Por otra parte, la cocaína induce un estado protrombótico mediante la estimulación de plaquetas y de la coagulación; con el tiempo, estos diversos efectos promueven aterosclerosis y disfunción endotelial, que aumentan aún más el riesgo de eventos cardiovasculares en los consumidores crónicos de cocaína ${ }^{44}$; adicionalmente, esta bloquea los canales de $\mathrm{Na}^{+}$en las membranas, cambiando el potencial de acción asociado a su efecto anestésico local ${ }^{44,45}$.

- Cardiopatía de takotsubo por alcohol (5/38 casos equivalente al $13,2 \%)^{22-26}$ : la abstinencia alcohólica aguda puede causar un estado hiperadrenérgico durante 48 a 96 horas, lo que puede desencadenar temblor, ansiedad, náuseas, vómitos, diarrea, taquicardia e hipertensión ${ }^{23}$. Adicionalmente, existen varias explicaciones para la disminución de la contractilidad y la función ventricular causada por el alcohol: a) Daño mitocondrial, directamente por el alcohol o por la ausencia de la enzima alcohol deshidrogenasa en los cardiomiocitos; b) Lesión de los cardiomiocitos, reducción de la síntesis proteica e inhibición de la ATPasa miofibrilar, atribuidas al acetaldehído (metabolito del metabolismo del alcohol); c) Alteración de enzimas del ciclo del ácido tricarboxílico (ciclo de Krebs) y, con ello, inhibición de la cadena respiratoria; y d) Deterioro de la absorción y unión del calcio en la mitocondria ${ }^{45-49}$.

- Cardiopatía de takotsubo por venenos de origen animal $(5 / 38 \text { casos equivalente al } 13,2 \%)^{27-31}$ : los casos de miocardiopatía de takotsubo asociados a la picadura de un animal podrían ser explicados desde dos perspectivas. La primera supone que una exposición accidental es por sí misma un evento estresante; y la segunda sugiere que hay evidencia de que algunos de los venenos son estimulantes químicos directos que causan disfunción cardiaca, como es el caso del veneno de la araña viuda negra, que contiene alfa-latrotoxina, la cual induce la liberación de neurotransmisores desde las terminales nerviosas ${ }^{29}$; el veneno del escorpión, que contiene varias toxinas, en especial alfa-toxinas, las cuales tienen la propiedad de inducir una estimulación neuronal, principalmente en el centro autonómico, provocando la liberación de catecolaminas hacia la circulación ${ }^{10,11,31}$; y el veneno de medusa, que causa daño cardiaco, el cual hace parte de los síntomas del "síndrome Irukandji",', que está asociado a un exceso de liberación de catecolaminas endógenas ${ }^{27,28}$.

- Cardiopatía de takotsubo por anfetaminas (4/38 casos equivalente al $10,5 \%)^{32-35}$ : la 4-fluoroanfetamina (4-FA) inhibe la recaptación y estimula la liberación de dopamina, serotonina y norepinefrina. En comparación con otras anfetaminas halogenadas, 4-FA tiene un efecto más débil, de corta duración y reversible en la disminución de serotonina y en la inhibición de la monoamina oxidasa ${ }^{32}$.

- Cardiopatía de takotsubo por el hongo Psilocybe semilanceata $(1 / 38 \text { casos equivalente al } 2,6 \%)^{36}$ : este hongo posee un metabolito que interactúa con receptores dopaminérgicos, adrenérgicos y serotoninérgicos, causando disfunción contráctil del miocardio. Los hongos de este tipo contienen triptamina, profármaco de la psilocina, una sustancia que, además de sus efectos simpaticomiméticos deletéreos sobre el miocárdico ${ }^{36}$, se comporta como un agonista parcial de los receptores serotoninérgicos 5HT1A y 5-HT2A, lo que puede desencadenar un espasmo de la arteria coronaria.

- Cardiopatía de takotsubo por marihuana (1/38 casos equivalente al 2,6\% $)^{37}$ : los endocannabinoides pueden causar una disminución de la contractilidad y aturdimiento miocárdico debido a su capacidad de estimular receptores CB-1. Un mecanismo similar podría aplicarse a los cannabinoides exógenos, tales como la marihuana, cuyo consumo agudo causa un aumento de la actividad del sistema nervioso simpático.

- Cardiopatía de takotsubo por abstinencia de heroína $(1 / 38 \text { casos equivalente al } 2,6 \%)^{38}$ : aunque la heroína aumenta la actividad parasimpática y disminuye la simpática, en los casos se abstinencia a la droga se produce un estado hiperadrenérgico y de irritabilidad del sistema nervioso central, lo que puede explicar el aumento en los niveles de catecolaminas y sus efectos a nivel cardiaco ${ }^{50}$.

- Cardiopatía de takotsubo por insecticida (1/38 casos equivalente al $2,6 \%)^{39}$ : la exposición a carbamatos y piretroides puede causar daños cardiacos por su toxicidad; además estos productos aumentan los niveles de acetilcolina y, con ello, sus efectos colinérgicos y muscarínicos ${ }^{39}$.

- Cardiopatía de takotsubo por cloroquina (1/38 casos equivalente al $2,6 \%)^{40}$ :la dosis excesiva de cloroquina provoca una liberación adrenérgica endógena intensa en respuesta al bloqueo de la conducción ventricular ${ }^{40}$.

- Cardiopatía de takotsubo por bebidas energéticas (1/38 casos equivalente al 2,6\%) $)^{41}$ : este tipo de bebidas contiene sustancias simpaticomiméticas, tales como cafeína y 1,3-dimetilamilamina (DMAA). La cafeína es un agonista competitivo de los receptores de adenosina A1 y A2, tanto del sistema nervioso central como del miocardio, que altera la liberación de neurotransmisores y la frecuencia cardiaca. Adicionalmente, provoca un aumento de calcio intracelular en los miocitos y, con ello, posibles alteraciones en el ritmo cardiaco. Por su parte, la DMAA, sustancia psicoactiva no aprobada por la Administración de Drogas y Alimentos (FDA, su sigla en inglés), puede causar un aumento de los niveles de adrenalina ${ }^{41}$. 
Con relación a la miocardiopatía de takotsubo es importante mencionar que, en 1991, Kounis et al. ${ }^{51}$ describieron el síndrome de la angina alérgica, en el cual la exposición a venenos de origen animal, químicos, factores ambientales, entre otros, se asocia con la aparición de eventos coronarios, acompañados de reacciones anafilácticas y anafilactoides. Los autores sugieren que la histamina, liberada durante las reacciones alérgicas, puede producir espasmo arterial coronario $\mathrm{y}$, con ello, angina de pecho debido a la estimulación de receptores $\mathrm{H}_{1}$ y $\mathrm{H}_{2}$ cardiacos y coronarios. En este sentido, se debe tener presente que algunos de los síntomas como la angina y la disnea, también se presentan en la miocardiopatía de takotsubo, lo que podría llevar a confusiones diagnósticas. Por ello, es necesario que el médico identifique y diferencie de manera correcta esta entidad de otros eventos coronarios y, con ello, prevenga a los pacientes de la exposición a posibles efectos adversos de las medidas terapéuticas utilizadas en el tratamiento de los eventos coronarios agudos. Resulta clave, así mismo, la identificación y caracterización de posibles desencadenantes, diferentes a los estados de estrés físico o emocional. En este contexto, esta revisión se convierte en un aporte relevante, debido a que identificó de forma estructurada, casos de miocardiopatía de takotsubo asociados con el uso o exposición de 14 drogas de abuso, sustancias químicas o venenos de origen animal. Ante la sospecha de un evento coronario agudo, el clínico debería indagar por el consumo o exposición a estos 14 productos, especialmente en quienes sea más probable dicha característica. De igual forma, los resultados de la revisión se convierten en un argumento a favor de la hipótesis del estado hiperadrenérgico directo o indirecto como mecanismo fisiopatológico de la miocardiopatía de takotsubo, tal como se aprecia en la mayoría de los 38 casos identificados.

\section{Limitaciones}

Los resultados de esta revisión deben interpretarse con cautela debido a algunas limitaciones. En primer lugar, la información sólo fue obtenida de los casos reportados, en los que no siempre se establece la existencia de una relación causal; y en segundo lugar, en la búsqueda solo se usó la base de datos PubMed/Medline, si bien, en general, en este tipo de trabajos se recomienda realizar la búsqueda en dos o más bases de datos. Sin embargo, esta limitación pudo ser disminuida debido a la búsqueda de otros reportes en las referencias de los artículos seleccionados. Adicionalmente, aunque la hipótesis planteada sobre la aparición de miocardiopatía de takotsubo es apoyada por el mecanismo farmacológico y biológico de las sustancias en estudio, clínicamente las pruebas son limitadas. En consecuencia, los resultados actuales requieren más investigaciones diseñadas para evaluar el riesgo real de miocardiopatía de takotsubo inducida por drogas y mejorar el conocimiento de la fisiopatología de este tipo de miocardiopatía.

\section{Conclusiones}

La miocardiopatía de takotsubo podría ser desencadena por la utilización o exposición de 14 drogas de abuso, sustancias químicas o venenos de origen animal, las cuales por su mecanismo de acción, o por la respuesta física en asuntos de abstinencia, en la mayoría de los casos, generan de forma directa o indirecta una sobreestimulación simpática. Por ello, se recomienda considerar este tipo de situación en pacientes que presenten miocardiopatía, especialmente en quienes no se identifique de forma precisa un desencadenante físico o emocional, en quienes exista sospecha o certeza del consumo de drogas de abuso como cocaína, heroína y anfetaminas, y en quienes se ha reportado exposición a venenos de origen animal o al monóxido de carbono.

\section{Responsabilidades éticas}

Protección de personas y animales. Los autores declaran que para esta investigación no se han realizado experimentos en seres humanos ni en animales.

Confidencialidad de los datos. Los autores declaran que en este artículo no aparecen datos de pacientes.

Derecho a la privacidad y consentimiento informado. Los autores declaran que en este artículo no aparecen datos de pacientes.

\section{Conflictos de interés}

Los autores declaran no tener conflictos de interés.

\section{Agradecimientos}

Al Comité para el Desarrollo de la Investigación (CODI) de la Universidad de Antioquia, por su apoyo económico en la convocatoria sostenibilidad (2014 a 2015), al grupo Promoción y Prevención Farmacéutica y al Programa Joven Investigador Colciencias-Universidad de Antioquia, que posibilita la participación y formación de la química farmacéutica Laura Cifuentes.

\section{Bibliografía}

1. Aizawa K, Suzuki T. Takotsubo cardiomyopathy: Japanese perspective. Heart Fail Clin. 2013;9(2):243-7.

2. Scantlebury DC, Prasad A. Diagnosis of Takotsubo Cardiomyopathy. Circ J. 2014;78(9):2129-39.

3. Kurisu S, Inoue I, Kawagoe T, Ishihara M, Shimatani Y, Nakama $Y$, et al. Prevalence of incidental coronary artery disease in tako-tsubo cardiomyopathy. Coron Artery Dis. 2009;20(3): 214-8.

4. Santoro F, leva R, Musaico F, Ferraretti A, Triggiani G, Tarantino $\mathrm{N}$, et al. Lack of efficacy of drug therapy in preventing Takotsubo cardiomyopathy recurrence: a meta-analysis. Clin Cardiol. 2014;37(7):434-9.

5. Redfors B, Shao Y, Omerovic E. Stress-induced cardiomyopathy in the critically ill - why inotropes fail to improve outcome. Int J Cardiol. 2013;168(4):4489-90.

6. Kurisu S, Inoue I, Kawagoe T, Ishihara M, Shimatani Y, Nakama $\mathrm{Y}$, et al. Presentation of Tako-tsubo cardiomyopathy in men and women. Clin Cardiol. 2010;33(1):42-5.

7. Amariles P. A comprehensive literature search: drugs as possible triggers of takotsubo cardiomyopathy. Curr Clin Pharmacol. 2011;6:1-11. 
8. Chung H, Kwon SW, Kim TH, Yoon JH, Ma DW, Park YM, et al. Synephrine-containing dietary supplement precipitating apical ballooning syndrome in a young female. Korean J Intern Med. 2013;28(3):356-60.

9. Jairam A, Kumar RS, Ghosh AK, Hasija PK, Singh JI, Mahapatra D, et al. Delayed Kounis syndrome and acute renal failure after wasp sting. Int J Cardiol. 2010;138(1):e12-4.

10. Miranda CH, Braggion-Santos MF, Schmidt A, PazinFilho A, Cupo P. Evolution of the electrocardiogram QRS complexes voltage in scorpion envenomation-induced Takotsubo syndrome. Am J Emerg Med. 2015;33(6):837-8. «Carta».

11. Madias JE. Voltage attenuation of the electrocardiogram QRS complexes in a patient with scorpion envenomationinduced Takotsubo syndrome. Am J Emerg Med. 2015;33(6):838. «Carta».

12. Ungprasert P, Srivali N. Electrocardiogram changes and prognosis of takotsubo cardiomyopathy. Am J Emerg Med. 2015;33(5):724-5.

13. Madias JE. Any particulars in the electrocardiogram of patients with basal Takotsubo syndrome? Int J Cardiol. 2014;172(1):e59.

14. Stohs SJ. Unsupported conclusions in the article Synephrinecontaining dietary supplement precipitating apical ballooning syndrome in a young female. Korean J Intern Med. 2014;29(3):388-92. «Carta».

15. Jung YS, Lee JS, Min YG, Park JS, Jeon WC, Park EJ, et al. Carbon monoxide-induced cardiomyopathy. Circ J. 2014;78(6):1437-44.

16. Serrão MG, Nascimento R, Santos N, Pereira A, Barros A, Pereira D, et al. Transient cardiomyopathy secondary to carbon monoxide poisoning. Rev Port Cardiol. 2008;27(6):833-8.

17. Daka MA, Khan RS, Deppert EJ. Transient left ventricular apical ballooning after a cocaine binge. J Invasive Cardiol. 2007;19(12):E378-80.

18. Sarkar S, Arguelles E, de Elia C. Takotsubo cardiomyopathy presenting as a non-ST segment elevation myocardial infarction in the setting of cocaine use and asthma exacerbation. Int $J$ Cardiol. 2013;168(1):e1-2.

19. Arora S, Alfayoumi F, Srinivasan V. Transient left ventricular apical ballooning after cocaine use: is catecholamine cardiotoxicity the pathologic link? Mayo Clin Proc. 2006;81(6): 829-32.

20. Butterfield M, Riguzzi C, Frenkel O, Nagdev A. Stimulant-related Takotsubo cardiomyopathy. Am J Emerg Med. 2015;33(3):476, e1-3.

21. Chokshi SK, Moore R, Pandian NG, Isner JM. Reversible cardiomyopathy associated with cocaine intoxication. Ann Intern Med. 1989;111(12):1039-40.

22. Alexandre J, Benouda L, Champ-Rigot L, Labombarda F. Takotsubo cardiomyopathy triggered by alcohol withdrawal. Drug Alcohol Rev. 2011;30(4):434-7.

23. Stout BJ, Hoshide R, Vincent DS. Takotsubo cardiomyopathy in the setting of acute alcohol withdrawal. Hawaii J Med Public Health. 2012;71(7):193-4.

24. Omar HR, Abdelmalak HD, Komorova I, Helal E, Camporesi EM. Alcohol withdrawal-induced Takotsubo. Intern Emerg Med. 2012;7 Suppl 2:S107-8.

25. Suzuki K, Osada N, Akasi YJ, Suzuki N, Sakakibara M, Miyake $\mathrm{F}$, et al. An atypical case of Takotsubo cardiomyopathy during alcohol withdrawal: abnormality in the transient left ventricular wall motion and a remarkable elevation in the ST segment. Intern Med. 2004;43(4):300-5.

26. Roy RR, Hakim FA, Hurst RT, Simper D, Appleton CP. Two cases of apical ballooning syndrome masking apical hypertrophic cardiomyopathy. Tex Heart Inst J. 2014;41(2):179-83.

27. Bianchi R, Torella D, Spaccarotella C, Mongiardo A, Indolfi C. Mediterranean jellyfish sting-induced Tako-Tsubo cardiomyopathy. Eur Heart J. 2011;32(1):18.
28. Tiong K. Irukandji syndrome, catecholamines, and midventricular stress cardiomyopathy. Eur J Echocardiogr. 2009;10(2):334-6.

29. Alexakis L, Arapi S, Stefanou I, Gargalianos P, Astriti M. Transient reverse takotsubo cardiomyopathy following a spider bite in Greece: a case report. Medicine (Baltimore). 2015;94(5):e457.

30. Murase K, Takagi K. Takotsubo cardiomyopathy in a snake bite victim: a case report. Pan Afr Med J. 2012;13:51.

31. Miranda CH, Braggion-Santos MF, Schmidt A, Pazin-Filho A, Cupo P. The first description of cardiac magnetic resonance findings in a severe scorpion envenomation: Is it a stress-induced (Takotsubo) cardiomyopathy like? Am J Emerg Med. 2015;33(6):862, e5-7.

32. Al-Abri S, Meier KH, Colby JM, Smollin CG, Benowitz NL. Cardiogenic shock after use of fluoro amphetamine confirmed with serum and urine levels. Clin Toxicol (Phila). 2014;52(10):1292-5.

33. Fulcher J, Wilcox I. Basal stress cardiomyopathy induced by exogenous catecholamines in younger adults. Int J Cardiol. 2013;168(6):e158-60.

34. Srikanth S, Barua R, Ambrose J. Methamphetamine-associated acute left ventricular dysfunction: a variant of stress-induced cardiomyopathy. Cardiology. 2008;109(3):188-92.

35. Movahed MR, Mostafizi K. Reverse or inverted left ventricular apical ballooning syndrome (reverse Takotsubo cardiomyopathy) in a young woman in the setting of amphetamine use. Echocardiography. 2008;25(4):429-32.

36. Nef HM, Möllmann H, Hilpert P, Krause N, Troidl C, Weber M, et al. Apical regional wall motion abnormalities reminiscent to Tako-Tsubo cardiomyopathy following consumption of psychoactive fungi. Int J Cardiol. 2009;134(1):e39-41.

37. Nogi M, Fergusson D, Chiaco JM. Mid-ventricular variant Takotsubo cardiomyopathy associated with Cannabinoid Hyperemesis Syndrome: a case report. Hawaii J Med Public Health. 2014;73(4):115-8.

38. Revelo AE, Pallavi R, Espana-Schmidt C, Visco F, Pekler G, Mushiyev S. Stoned people can get stunned myocardium: a case of heroin withdrawal precipitating Tako-Tsubo cardiomyopathy. Int J Cardiol. 2013;168(3):e96-8.

39. Lin CC, Lai SY, Hu SY, Tsan YT, Hu WH. Takotsubo cardiomyopathy related to carbamate and pyrethroid intoxication. Resuscitation. 2010;81(8):1051-2.

40. Champion S, Malissin I, Cleophax C, Vodovar D, Deye $\mathrm{N}$, Mégarbane $\mathrm{B}$, et al. Chloroquine poisoning-associated inverted Tako-tsubo cardiomyopathy. Clin Toxicol (Phila). 2012;50(7):721-2.

41. Kaoukis A, Panagopoulou V, Mojibian HR, Jacoby D. Reverse Takotsubo cardiomyopathy associated with the consumption of an energy drink. Circulation. 2012;125(12):1584-5.

42. Castaño G, Calderón G. Consumo de heroína en Colombia, prácticas relacionadas e incidencia en la salud pública. Rev Cub Salud Publica. 2010;36(4):11-322.

43. Tranter MH, Wright PT, Sikkel MB, Lyon AR. Takotsubo cardiomyopathy: the pathophysiology. Heart Fail Clin. 2013;9(2):187-96.

44. Liaudet L, Calderari B, Pacher P. Pathophysiological mechanisms of catecholamine and cocaine-mediated cardiotoxicity. Heart Fail Rev. 2014;19(6):815-24.

45. Figueredo VM. Chemical cardiomyopathies: the negative effects of medications and non-prescribed drugs on the heart. Am J Med. 2011;124(6):480-8.

46. Finsterer J, Ohnsorge P. Influence of mitochondrion-toxic agents on the cardiovascular system. Regul Toxicol Pharmacol. 2013;67(3):434-45.

47. Lucas DL, Brown RA, Wassef M, Giles TD. Alcohol and the cardiovascular system: research challenges and opportunities. J Am Coll Cardiol. 2005;45(12):1916-24. 
48. de Leiris J, de Lorgeril M, Boucher F. Ethanol and cardiac function. Am J Physiol Heart Circ Physiol. 2006;291(3):H1027-8.

49. Walker RK, Cousins VM, Umoh NA, Jeffress MA, Taghipour D, Al-Rubaiee $M$, et al. The good, the bad, and the ugly with alcohol use and abuse on the heart. Alcohol Clin Exp Res. 2013;37(8):1253-60.
50. Sarcon A, Ghadri JR, Wong G, Lüscher TF, Templin C, Amsterdam E. Takotsubo cardiomyopathy associated with opiate withdrawal. QJM. 2014;107(4):301-2.

51. Kounis NG, Zavras GM. Histamine-induced coronary artery spasm: the concept of allergic angina. $\mathrm{Br} \mathrm{J}$ Clin Pract. $1991 ; 45(2): 121-8$. 